\title{
Aggregate Bandwidth Estimation in Stored Video Distribution Systems ${ }^{1}$ \\ G. Boggia, P. Camarda ${ }^{\circ}$ D. Striccoli \\ Politecnico di Bari - Dip. di Elettrotecnica ed Elettronica \\ Via E. Orabona, 4 - 70125 Bari (Italy) \\ E-mail: boggia@ieee.org; camarda@poliba.it; striccoli@deemail.poliba.it
}

\begin{abstract}
Multimedia applications like Video on Demand (VoD), Distance Learning, Internet Video Broadcast, etc., will play a fundamental role in future broadband networks. A common aspect of such applications is the transmission of video streams that require a sustained relatively high bandwidth with stringent requirements of Quality of Service (QoS). In this paper various original algorithms for evaluating, in a video distribution system, a statistical estimation of aggregate bandwidth needed by a given number of smoothed video streams are proposed and discussed. The VBR traffic generated by each video stream is characterized by its marginal distribution and by conditional probabilities between rates of temporary closed streams. The developed iterative algorithms evaluate an upper and lower bound of needed bandwidth for guaranteeing a given loss probability. The obtained results are compared with simulations and with other results, based on similar assumptions, already presented in the literature. Some considerations on the developed algorithms are made, in order to evaluate the effectiveness of the proposed methods.
\end{abstract}

Keywords: Video Distribution, Bandwidth Evaluation, Analytical and Simulation Model.

Corresponding Author

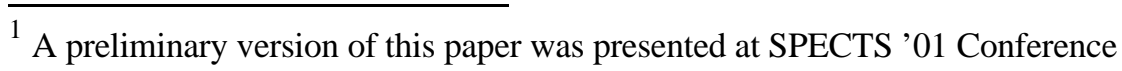




\section{INTRODUCTION}

Multimedia applications are expected to occupy a dominant role in future broadband telecommunication networks. The video and audio delivery among network users with specific Quality of Service (QoS) guarantees (maximum delay, loss probability, etc.), implies a detailed analysis of the properties and structure of transmitted data. In our study we will refer to video distribution systems, which include applications like Video on Demand (VoD), Distance Learning, Internet video broadcast, etc [1]. A common aspect of such applications is the transmission of video streams that require a sustained relatively high VBR bandwidth with a stringent Quality of Service (QoS) guarantee. An efficient delivery of the video streams to final users while sharing network resources, guaranteeing the needed QoS, requires specific Admission Control schemes [6, 7, 8]. These algorithms, which limit the total number of accepted video streams in the network, are based on a statistical estimation of the aggregate bandwidth needed by a certain number of video streams. Standard video coding techniques (e.g., MPEG, H323, etc.) produce a VBR traffic. As an example, in Figure 1a the bit rate of the first 32000 frames of an MPEG-1 coded trace of the film "Star Wars" is reported. As highlighted in $[8,9]$ a consistent gain in network resource utilization can be obtained by exploiting smoothing, which will probably be implemented in real systems. These algorithms reduce the peak rate and its variability of each stream present in the network using a transmission buffer that regularizes the bit flow. On the client side, the smoothed video stream is stored in a client buffer from which the original unsmoothed video stream is extracted, decoded and displayed. In Figure $1 \mathrm{~b}$ the bit rate of the film "Star Wars" smoothed with a client buffer of 1.0M Bytes is reported.

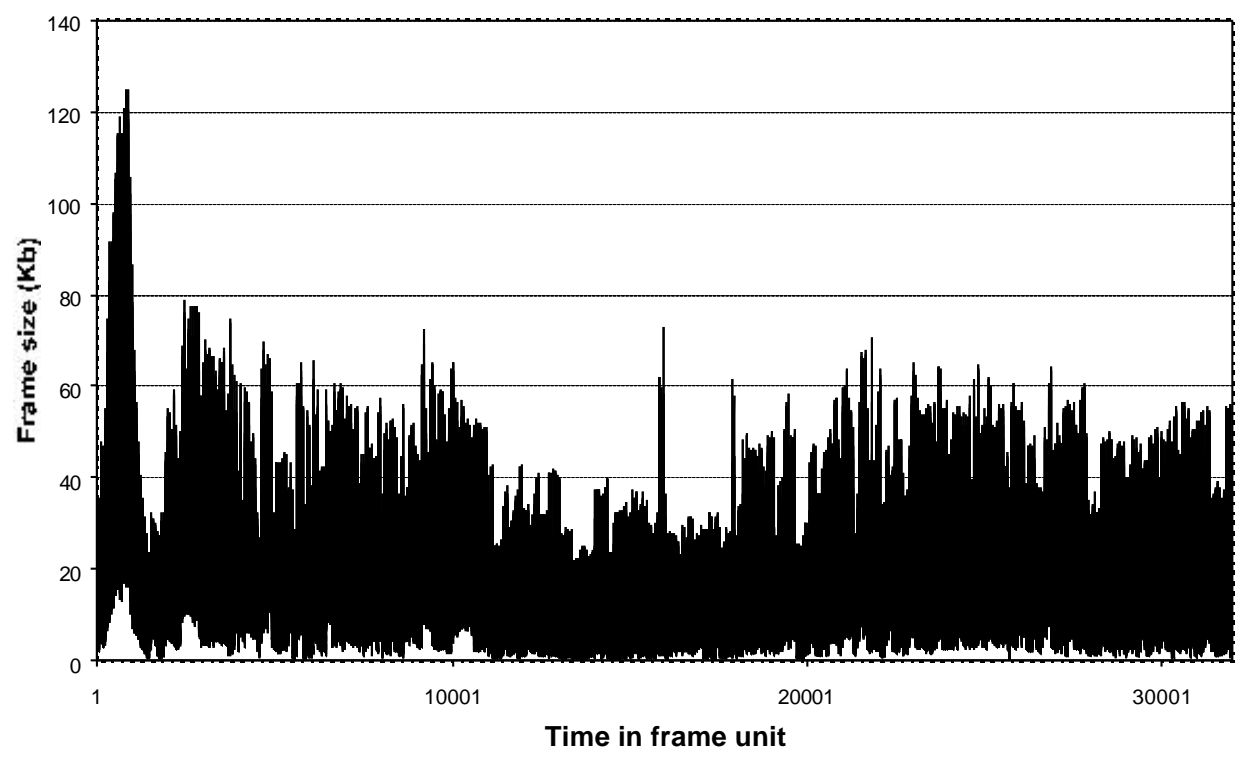

Figure 1a. 32000 frames of the film "Star Wars" without smoothing. 


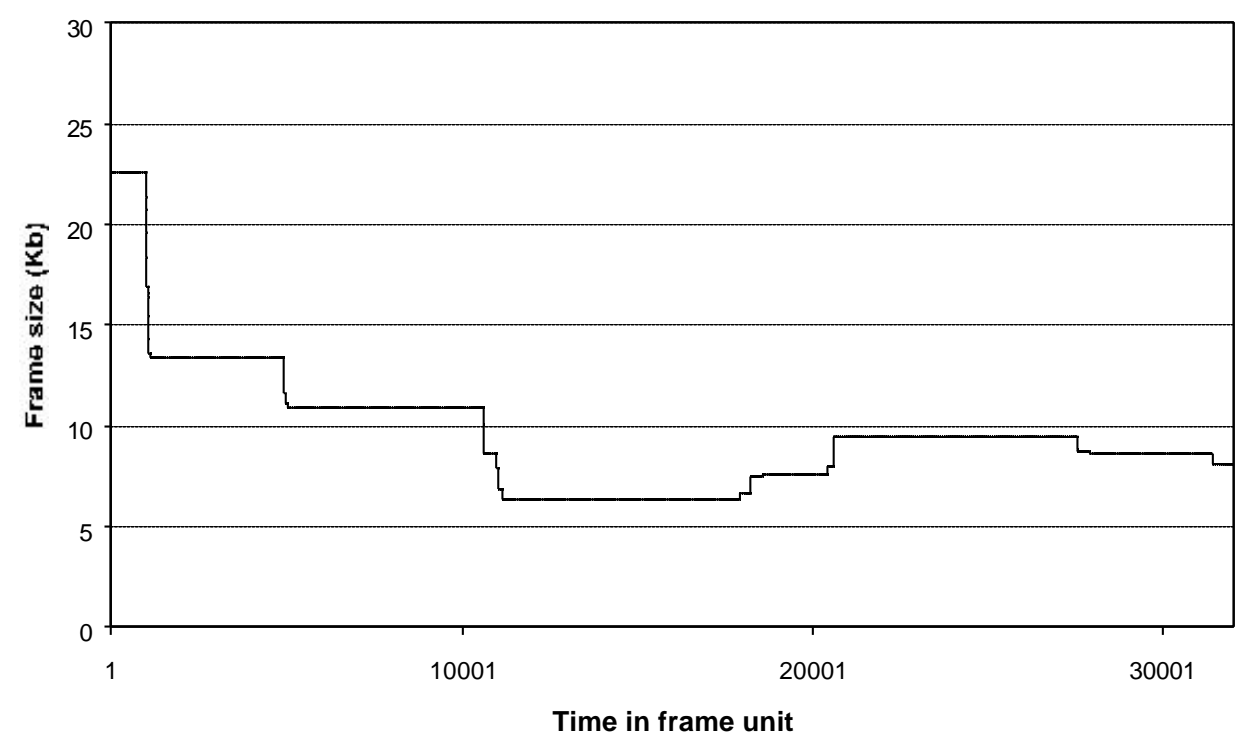

Figure 1b. 32000 frames of the film "Star Wars" with smoothing (buffer size = 1 MB).

As can be inferred, the smoothing technique consistently reduces the rate variability. The consequence of this technique is that the marginal distribution of bit rate in smoothed sequences is quite radically different when compared with original unsmoothed sequences. As an example of the alteration of the frame size distribution due to the smoothing algorithms, in Figures $2 a$ and $2 b$ the bit rate histograms relative to 40000 frames of the Star Wars film are reported. In Figure 2a the video stream is unsmoothed, while in Figure $2 b$ it is smoothed using a client buffer of $1 \mathrm{MB}$. Various smoothing techniques have been proposed in the literature $[9,10]$, nevertheless the study developed in this paper, as will be clear in the sequel, is valid whatever smoothing technique is exploited.

A significant problem in the considered systems is the aggregate bandwidth estimation needed for planning the transmission infrastructure and for admission control. Such a problem, in the literature, has been attacked by exploiting Chernoff bound, in the hypothesis of independence among video streams and bufferless systems. The last hypothesis has been taken into account in [8] and can be supported by the following considerations. First of all, it has been shown $[11,12]$ that large buffers are of substantial benefit in reducing losses in the high frequency domain and the only effective way to reduce losses in the low frequency domain is to allocate sufficient bandwidth for each network link. Since the smoothed traffic mainly has a slow time scale variability, the adoption of large network buffers is not useful in this scenario. A second consideration is that the employment of large buffers may introduce delays along the network nodes that can be intolerable for the correct delivery of video streams and thus cannot meet the QoS specifications. Another significant aspect 
must be taken into account in the analysis of these systems. In fact, several studies on the bursty nature of the Variable Bit Rate (VBR) video traffic that characterizes this kind of application highlight some important properties of compressed video sources. In particular, it has been shown $[2,3,4,5]$ that the MPEG video traffic exhibits properties of self-similarity and Long Range Dependence (LRD) that may require sophisticated analytical models to adequately take into account the network traffic behavior. However, in this case, small buffers must be utilized and, as discussed in [13], long range dependence does not have a predominant effect on VBR coded video. As a matter of fact, in this paper the network switch will be modeled as a bufferless multiplexer, without exploiting long range dependence techniques.

In this paper various original iterative algorithms are proposed allowing evaluation of the considered contexts of lower and upper bounds of the aggregate bandwidth needed by a given number of video streams with a defined loss probability constraint. The obtained results are compared with simulation results and where possible with other results already present in literature. The input data of our algorithms are marginal distribution and conditional probabilities of the bit rate, derived directly from video traces. To adequately describe the marginal distribution of the single video stream we need a model that is general enough to permit the description of a great range of video streams and also quite simple to manage. The histogram method satisfies both these requirements $[14,8,15]$.

The paper is structured as follows. In Section II various algorithms for estimating the aggregate bandwidth are proposed. In particular, in Section II.1 the case of homogeneous streams is considered, under the hypothesis of independence among streams. In Section II.2 we consider the case of heterogeneous streams, under the same hypothesis. In Section II.3 heterogeneous streams are considered, but under the assumption of a certain degree of correlation among streams. In Section III we will show some numerical results and, finally, in Section IV some conclusions on this work will be given. 


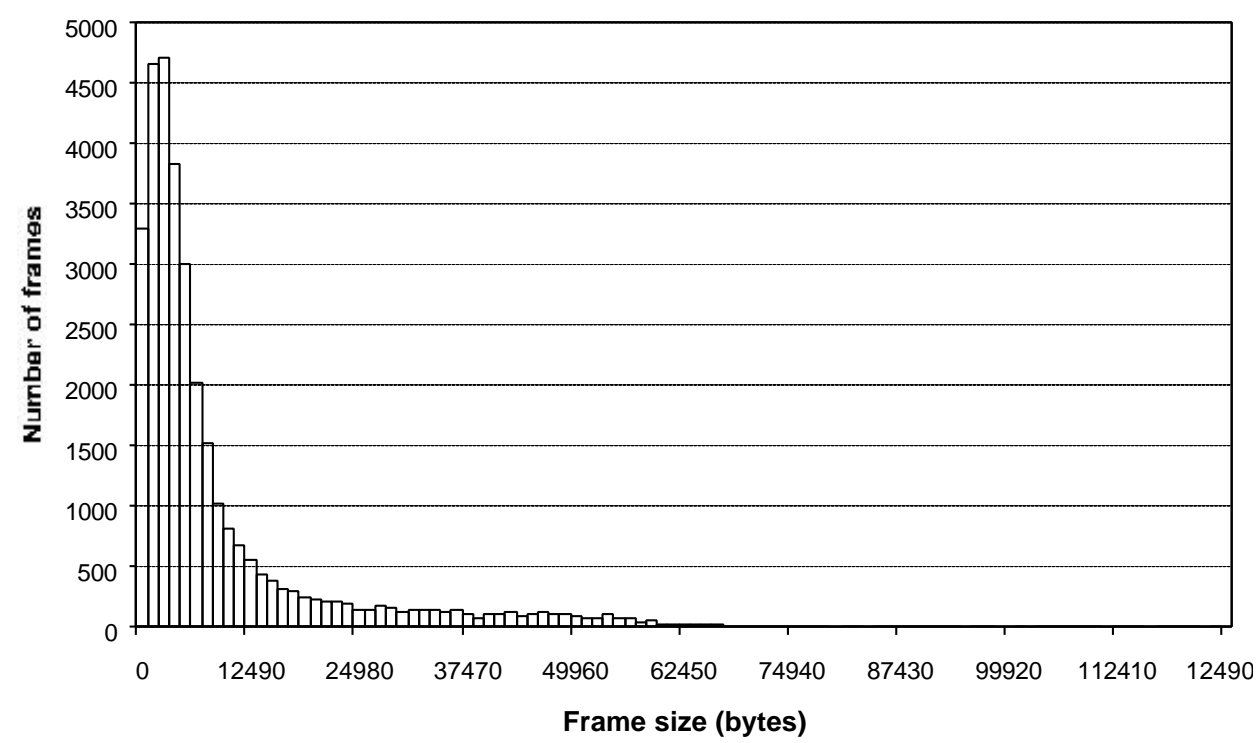

Figure 2a. Frame Size distribution for the film "Star Wars" without smoothing.

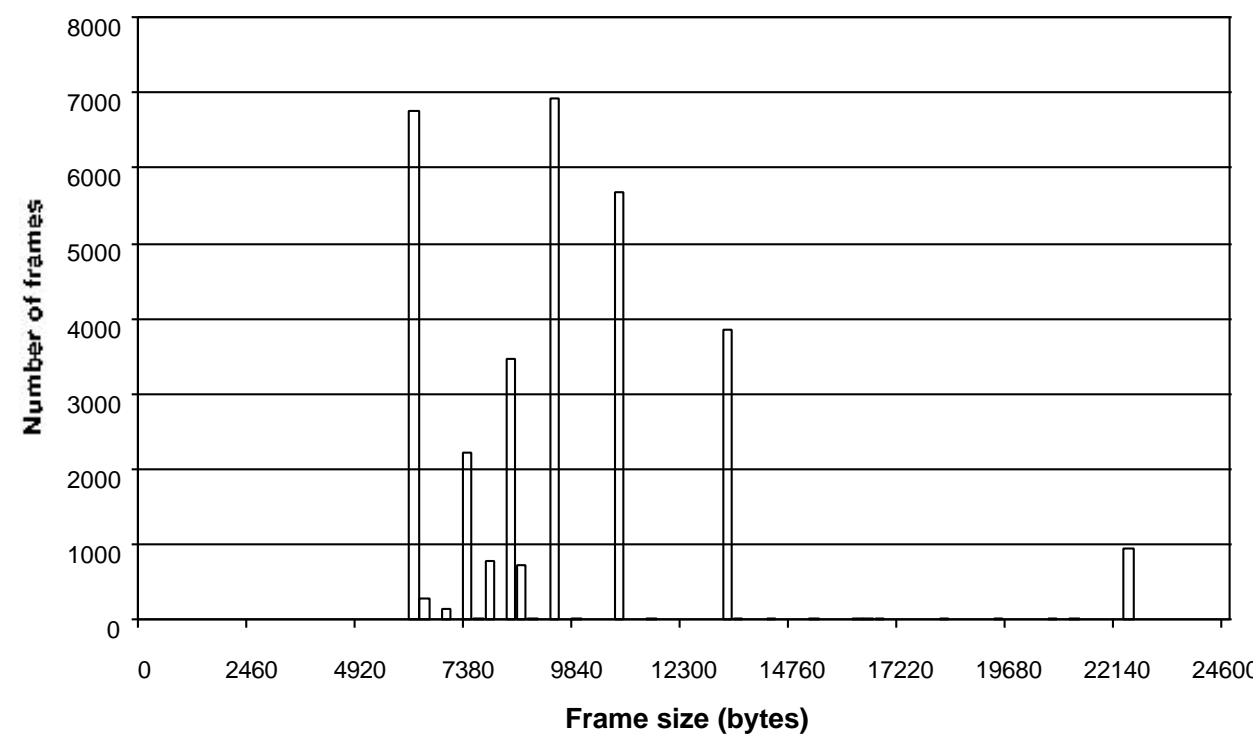

Figure 2b. Frame Size distribution for the film "Star Wars" with smoothing (buffer size 1 MB).

\section{AGGREGATE BANDWIDTH ESTIMATION}

In this section some methods to estimate the aggregate bandwidth needed by a generic number of video streams will be provided. Let us consider generically $\mathrm{N}$ video streams, either smoothed or unsmoothed, deriving from one or more sources. The bit rate marginal distribution of each stream is evaluated exploiting the histogram method, that consists in dividing the entire bit rate range of the single video stream in $\mathrm{K}$ equal bins. Then a discrete random variable that can assume $\mathrm{K}$ values $r_{1}<r_{2}<. .<r_{K}$ with probability $p\left(r_{i}\right)=p_{i}(i=1,2, \ldots K)$ is defined [8]. The $\mathrm{K}$ values are given by the upper extremes of each of the histogram bins. 
The system state can be described by the vector $\left(\alpha_{1}, \alpha_{2}, \ldots, \alpha_{N}\right)$, where the generic $\alpha_{i}$ $\left(\alpha_{i}=1,2, \ldots, K\right)$, identifies the $i^{\text {th }}$ stream rate. The corresponding state probability is given by the following quantity:

$$
p\left(\alpha_{1}, \alpha_{2}, \ldots, \alpha_{N}\right)
$$

Consequently the aggregate bandwidth occupied by the $\mathrm{N}$ video streams in this state will be:

$$
\Lambda=r_{\alpha_{1}}+r_{\alpha_{2}}+\ldots+r_{\alpha_{N}}
$$

Our purpose is to calculate the aggregate bandwidth, called $\Lambda_{S}$, that satisfies a given loss probability $p_{l}$ (that we suppose to be a QoS specification). We have

$$
\left\{\begin{array}{c}
P\left(\Lambda \leq \Lambda_{S}\right)=\left(1-p_{l}\right)=\sum_{\alpha_{1}=1}^{K} \ldots \sum_{\alpha_{N}=1}^{K} p\left(\alpha_{1}, \alpha_{2}, \ldots, \alpha_{N}\right) \\
r_{\alpha_{1}}+r_{\alpha_{2}}+\ldots+r_{\alpha_{N}} \leq \Lambda_{S}
\end{array}\right.
$$

i.e., the probability of having a bandwidth lower than $\Lambda_{S}$ is given by the sum of all the state probabilities so that the aggregate bandwidth is lower than $\Lambda_{S}$.

The general equation reported in (3) will be characterized and solved in three cases. In the first, considered in section II.1, the case of a single type of video stream under the hypothesis of independence of video streams will be analyzed. In the second case, analyzed in section II.2, different types of video streams, under the same hypothesis of independence of all streams will be taken into account. Finally, in the third case, developed in section II.3, a certain amount of correlation among different types of video streams will be considered. The analytical results are compared with simulation results. The simulations have been made considering generically $\mathrm{N}$ video streams. Each film is supposed to be transmitted in a cyclic way. To calculate the aggregate bandwidth corresponding to a defined loss probability (e.g. $10^{-6}$ ), the simulation experiment is repeated a number of times so that the loss probability is correctly estimated. In each simulation experiment the starting points of all the video streams are randomly displaced in a given time interval specified for each of the three cases mentioned above.

\section{II.1 BANDWIDTH ESTIMATION FOR HOMOGENEOUS STREAMS}

Let us suppose $\mathrm{N}$ independent video streams originating from the same source. Under these assumptions, we can express the state probability (1) in the following way:

$$
p\left(\alpha_{1}, \alpha_{2}, \ldots, \alpha_{N}\right)=\prod_{i=1}^{N} p_{\alpha_{i}}
$$


Furthermore, by considering that several streams can simultaneously exploit the same rate, the system state can be expressed, more simply, by the vector $\left(n_{1}, n_{2}, \ldots, n_{K}\right)$ where the generic $n_{i}$ indicates the number of streams that exploit rate $r_{i}$ Obviously, the total number of streams must be $\mathrm{N}$, i.e. $n_{1}+n_{2}+\ldots+n_{K}=N$. The needed bandwidth for this state is given by $n_{1} r_{1}+n_{2} r_{2}+\ldots+n_{K} r_{K}=\Lambda$. The state probability results

$$
p\left(n_{1}, \ldots, n_{K}\right)=\left(\begin{array}{l}
N \\
n_{1}
\end{array}\right)\left(\begin{array}{c}
N-n_{1} \\
n_{2}
\end{array}\right) . .\left(\begin{array}{c}
N-. .-n_{K-1} \\
n_{K}
\end{array}\right) \prod_{i=1}^{K} p^{{ }^{n i}}
$$

and so:

$$
p\left(n_{1}, \ldots, n_{K}\right)=N ! \prod_{i=1}^{K} \frac{p_{i}^{n i}}{n i !}
$$

This last result can be justified by simple combinatorial considerations, observing that the $n_{1}$ streams that exploit rate $r_{1}$ can occupy $n_{1}$ of the $\mathrm{N}$ streams and thus the number of combinations is $\left(\begin{array}{l}N \\ n_{1}\end{array}\right)$. Similarly the $n_{2}$ streams that exploit rate $r_{2}$, can occupy $n_{2}$ of the $N-n_{1}$ free streams and thus the number of combinations is $\left(\begin{array}{c}N-n_{1} \\ n_{2}\end{array}\right)$, etc.

The needed bandwidth can be evaluated only statistically. In particular, let $\Lambda_{S}$ be the available bandwidth; we have

$$
p(\Lambda \leq \Lambda s)=\sum_{n_{1}=0}^{N} \ldots \sum_{n_{K}=0}^{N} p\left(n_{1}, \ldots, n_{K}\right)
$$

with the constraints:

$$
\left\{\begin{array}{c}
n_{1}+n_{2}+\ldots+n_{k}=N \\
n_{1} r_{1}+n_{2} r_{2}+\ldots+n_{K} r_{K} \leq \Lambda_{S}
\end{array}\right.
$$

By imposing a uniform quantization in the histogram, i.e., $r_{2}=2 r_{1}, r_{3}=3 r_{1}, \ldots, r_{K}=K r_{1}$ the second constraint becomes $n_{1}+2 n_{2}+\ldots+K n_{K} \leq \Lambda_{S} / r_{1}$. The previous expression (6), including the constraints, can be synthetically written as:

$$
p(\Lambda \leq \Lambda s)=\sum_{\substack{n_{1}+n_{2}+\ldots+n_{K}=N \\ n_{l}+2 n_{2}+\ldots+K n_{K} \leq \Lambda_{s} / r}} N ! \prod_{i=1}^{K} \frac{p_{i}^{n_{i}}}{n_{i} !}
$$

It is quite intuitive to realize that the previous expression can be rewritten evaluating the number of terms required by the second relation of the constraints. We have 


$$
p\left(\Lambda \leq \Lambda_{S}\right)=\sum_{j=N}^{h}\left(\sum_{\substack{n_{l}+n_{2}+\ldots+n_{K}=N \\ n_{I}+2 n_{2}+\ldots+K n_{K}=j}} N ! \prod_{i=l}^{K} \frac{p_{i}^{n_{i}}}{n_{i} !}\right)
$$

where $h=\Lambda_{S} / r_{1}$.

The same expression can be obtained by a more formal (and much more complex) approach [16]. It is evident that when $\Lambda_{S}$ is equal to the maximum possible rate for $\mathrm{N}$ streams, i.e., $\Lambda_{S}=N K r_{1}$, the previous probability must be one.

A brute force evaluation of the previous expression is practically impossible, due to the exponential number of terms in the summation. With the aim of finding a simple iterative method, let us rewrite the previous expression (8) indicating the inner summation by the symbol $a_{j-N}$. We have:

$$
p\left(\Lambda \leq \Lambda_{S}\right)=\left(\sum_{j=N}^{h} a_{j-N}\right)
$$

Two alternative iterative algorithms are proposed in the sequel for evaluating these terms $a_{j-N}$. The simple iteration of the first proposed method is reported below (see appendix A for further details).

$$
p_{1} a_{j}=\sum_{i=1}^{K-1}\left[\frac{(N+1) i}{j}-1\right] p_{i+1} a_{j-i}
$$

where

$$
\left\{\begin{array}{c}
j=1, \ldots,(K-1) N \\
a_{j-i}=0 ; \quad j-i<0
\end{array}\right.
$$

with initial condition $a_{0}=p_{1}{ }^{N}$.

The terms $a_{j}$ have a precise physical meaning. In fact, $a_{0}$ represents the probability that all $\mathrm{N}$ streams are using their smallest bit rate, $r_{l}$, and thus the global bit rate is $N r_{l}$. Likewise, the term $a_{1}$ represents the probability that $\mathrm{N}-1$ streams exploit rate $r_{1}$, while one stream transmits using rate $r_{2}=2 r_{1}$, thus the needed bandwidth is $(N+1) r_{1}$. In general we can say that the term $a_{j}$ represents the probability that the $\mathrm{N}$ streams globally exploit a bandwidth given by $(N+j) r_{l}$.

As mentioned before, the sum of all $a_{j}$ must be one $\left(\sum_{j=0}^{(K-1) N} a_{j}=1\right)$, indeed, we have verified this condition in the numerical examples. The probability $p\left(\Lambda \leq \Lambda_{S}\right)$ in (9) can be evaluated by summing all values of $a_{j}$ for $j$ varying from $O$ to $h-N$. Alternatively, we may evaluate 
$1-p\left(\Lambda \leq \Lambda_{S}\right)$, by summing the values of $a_{j}$ for $j$ varying from $h-N+1$ to its maximum value $(K-1) N$.

The previous iterative expressions, very simple and efficient, present some problems of error propagation when the number of streams exceeds a few hundred or even less in some limited cases. To overcome this problem and thus generalize the solution, a new iterative expression is proposed below. We have to calculate:

$$
a_{j}=\sum_{\substack{n_{1}+n_{2}+\ldots+n_{K}=N \\ n_{1}+2 n_{2}+\ldots+K n_{K}=j}} N ! \prod_{r=1}^{K} \frac{p_{r}^{n_{r}}}{n_{r} !}, \quad \text { for } N \leq j \leq K N
$$

Now we define the following function:

$$
g_{s, j}(n)=\sum_{\substack{n_{1}+n_{2}+\ldots+n_{s}=n \\ n_{1}+2 n_{2}+\ldots+s n_{s}=j}} n ! \prod_{r=1}^{s} \frac{p_{r}^{n_{r}}}{n_{r} !}
$$

It is obvious that $a_{j}=g_{K, j}(N)$. We can also note that the generic function $g_{s, j}(n)$ will contain all the contributions relative to the function $g_{s-1, j}(n)$. The terms that must be added to those contained in $g_{s-1, j}(n)$ consider all conditions on $n_{s}$, with $n_{s}$ assuming all values between 0 and $N$. In particular, for $n_{s}=0$ we have:

$$
\left.g_{s, j}(n)\right|_{n_{s}=0}=\sum_{\substack{n_{1}+\ldots+n_{s-1}=n \\ n_{1}+\ldots+(s-1) n_{s-1}=j}} n ! \prod_{r=1}^{s} \frac{p_{r}^{n_{r}}}{n_{r} !}=g_{s-1, j}(n)
$$

for $n_{s}=1$ we obtain:

$$
\left.g_{s, j}(n)\right|_{n_{s}=1}=\sum_{\substack{n_{1}+\ldots+n_{s-1}=n-1 \\ n_{1}+\ldots+(s-1) n_{s-1}=(j-s)}} n ! p_{s} \prod_{r=1}^{s-1} \frac{p_{r}{ }^{n_{r}}}{n_{r} !}
$$

and finally:

$$
\left.g_{s, j}(n)\right|_{n_{s}=1}=\left(\frac{n !}{(n-1) !} p_{s}\right) g_{s-1, j-s}(n-1)
$$

Following the same procedure we find that, in general:

$$
\left.g_{s, j}(n)\right|_{n_{s}=q}=\left(\frac{n !}{(n-q) !} \frac{p_{s}{ }^{q}}{q !}\right) g_{s-1, j-q s}(n-q), \quad \text { for } 0 \leq q \leq \frac{j-n}{s-1}
$$

The parameter $q$ can assume values up to the integer part of $\frac{j-n}{s-1}$. In fact, for the generic $g_{s, j}(n)$, it must be verified that $j \geq n$. Similarly, for the function $g_{s-1, j-q s}(n-q)$ we have $j-q s \geq n-q$. 
If now we call: $I=\operatorname{int}\left(\frac{j-n}{s-1}\right)$, we have:

$$
g_{s, j}(n)=\left.\sum_{p=0}^{I} g_{s, j}(n)\right|_{n_{s}=p} .
$$

For this reason:

$$
g_{s, j}(n)=\sum_{q=0}^{I}\left(\frac{n !}{(n-q) !} \frac{p_{s}^{q}}{q !}\right) g_{s-1, j-q s}(n-q)
$$

The equation (13) has to be used for $s=1,2, \ldots K$ and for $n=1,2, \ldots N$. The initial conditions are:

$$
\begin{array}{ll}
g_{s, 0}(0)=1, & s=1,2, \ldots K \\
g_{1, n}(n)=p_{1}{ }^{n}, & n=1,2, \ldots N
\end{array}
$$

Iterating in this way, we finally obtain all the functions $g_{K, j}(N)$ for each $j$, corresponding to all needed terms $a_{j}$.

\section{II.2 BANDWIDTH ESTIMATION FOR HETEROGENEOUS STREAMS}

In this section the aggregate bandwidth in the case of streams that have different statistical characteristics (as usual with variegated sources), will be evaluated exploiting the results of the previous section. We suppose that the video server has $M$ different types of films. Each one is characterized by the histogram method, that consists in defining, for a generic film $i$, a discrete r.v. that can assume the $K_{i}$ values $r_{i 1}<r_{i 2}<r_{i k_{i}}$ with probability $p\left(r_{i j}\right)=p_{i j} \quad\left(j=1, \ldots, K_{i}\right)$. We suppose that a user can choose a film $i$ with probability $\beta_{i}$ (obviously, $\beta_{1}+\beta_{2}+\ldots+\beta_{M}=1$ ).

In this case, the generic term $\alpha_{i}$ of the vector $\left(\alpha_{1}, \alpha_{2}, \ldots \alpha_{N}\right)$ is composed by a couple of indices $(i, j)$ that identify the type of stream and the relative rate. In the simplifying hypothesis that the $N$ video streams are independent, the state probability assumes the expression (4) and the corresponding aggregate bandwidth will be given by (2).

By considering that several streams can simultaneously exploit the same rate, the system state can be expressed, more simply, by $\left(n_{11}, n_{12}, \ldots, n_{1 k_{1}}, n_{21}, \ldots, n_{M k_{M}}\right)$ where the generic $n_{i j}$ indicates the number of type $i$ streams that exploit the rate $r_{i j}$. Obviously, the total number of streams must be N. The needed bandwidth for this state is given by:

$$
\Lambda=n_{11} r_{11}+n_{12} r_{12}+\ldots+n_{1 k_{1}} r_{1 k_{1}}+\ldots+n_{M k_{M}} r_{M k_{M}} .
$$

Following the same concepts developed for the single type of stream, the state probability results 


$$
p\left(n_{11}, . ., n_{M k_{M}}\right)=\left(\begin{array}{c}
N \\
n_{11}
\end{array}\right) \cdot\left(\begin{array}{c}
N-n_{11}-. .-n_{M, K_{M}-1} \\
n_{M K_{M}}
\end{array}\right) \prod_{m=1}^{M} \beta_{m}^{n_{m}} \prod_{r=1}^{K_{m}} p_{m r}^{n_{m r}}
$$

and, after some simple algebra:

$$
p\left(n_{11}, \ldots, n_{M k_{M}}\right)=N ! \prod_{m=1}^{M} \prod_{r=1}^{K_{m}} \frac{\left(\beta_{m} p_{m r}\right)^{n_{m r}}}{n_{m r} !}
$$

The needed aggregate bandwidth can be evaluated only statistically and in particular we have

$$
P\left(\Lambda \leq \Lambda_{S}\right)=\sum_{n_{I I}=0}^{N} \sum_{n_{12}=0}^{N} \ldots \sum_{n_{M K_{M}}=0}^{N} N ! \prod_{m=1}^{M} \prod_{r=1}^{K_{m}} \frac{\left(\beta_{m} p_{m r}\right)^{n_{m r}}}{n_{m r} !}
$$

with the constraints

$$
\left\{\begin{array}{c}
n_{11}+n_{12}+\ldots+n_{1 k_{l}}+\ldots+n_{M k_{M}}=N \\
n_{11} r_{11}+n_{12} r_{12}+\ldots+n_{1 k_{l}} r_{1 k_{l}}+\ldots+n_{M k_{M}} r_{M k_{M}} \leq \Lambda_{S}
\end{array}\right.
$$

By imposing the uniform quantization among all films, i.e.,

$$
\left\{\begin{array}{c}
r_{11}=r_{21}=\ldots=r_{M 1}=r_{1} \\
r_{12}=r_{22}=\ldots=r_{M 2}=r_{2}=2 r_{1} \\
\ldots \\
r_{1 K}=r_{2 K}=\ldots=r_{M K}=r_{K}=K r_{1}
\end{array}\right.
$$

and exploiting an approach similar to the homogeneous case, it is possible to find the following result [16]:

$$
\begin{gathered}
P\left(\Lambda \leq \Lambda_{S}\right)=\sum_{j=N}^{h} a_{j-N}, \quad h=\Lambda_{S} / r_{1} \\
a_{j-N}=\sum_{\substack{n_{11}+\ldots+n_{M K_{M}}=N \\
n_{11} r_{I I}+\ldots+n_{M K_{M}} r_{M K_{M}}=j}} N ! \prod_{m=1}^{M} \prod_{r=1}^{K_{m}} \frac{\left(\beta_{m} p_{m r}\right)^{n_{m r}}}{n_{m r} !} \quad, \quad N \leq j \leq K N
\end{gathered}
$$

The previous expression, like the homogeneous case, cannot be directly evaluated due to its complexity. Also in this case an iterative approach can be pursued. In particular we can find, exploiting the multinomial formula and equation (17), that:

$$
\begin{gathered}
a_{0}=\left(\sum_{m=1}^{M}\left(\beta_{m} p_{m 1}\right)\right)^{N} \\
a_{1}=N\left(\sum_{m=1}^{M} \beta_{m} p_{m 2}\right)\left[\left(\sum_{m=1}^{M} \beta_{m} p_{m 1}\right)^{N-1}\right]
\end{gathered}
$$

Indicating by

$$
p_{1}=\sum_{m=1}^{M}\left(\beta_{m} p_{m 1}\right) ; \quad p_{2}=\sum_{m=1}^{M} \beta_{m} p_{m 2}
$$


we have $a_{0}=p_{1}^{N}, a_{1}=N p_{2} p_{1}^{N-1}$, which correspond to the first two terms of the iterative method obtained in case of homogeneous streams.

In general:

$$
p_{j}=\sum_{m=1}^{M} \beta_{m} p_{m j} \quad \forall 1 \leq m \leq K
$$

With the previous position, the terms $a_{j-N}$ expressed by (17) become equivalent to the corresponding terms in (9) relative to the homogeneous case and thus a similar approach can be followed to find an iterative solution. In general, the following iterative algorithm can be found:

$$
p_{1} a_{j}=\sum_{i=1}^{K-1}\left[\frac{(N+1) i}{j}-1\right] p_{i+1} a_{j-i},
$$

where

$$
\left\{\begin{array}{c}
j=1, \ldots,(K-1) N \\
a_{j-i}=0 ; \quad j-i<0
\end{array}\right.
$$

with $a_{0}=p_{1}^{N}$. Equivalently, the same approach as the iterative method through the equation (13) can be used.

Also in this case, $a_{0}$ represents the probability that all $N$ streams are using their smallest bit rate, $r_{l}$, and thus the global bandwidth is $N r_{1}$. Likewise, the term $a_{1}$ represents the probability that $N-1$ streams are exploiting the rate $r_{1}$, while one stream is using the rate $r_{2}=2 r_{1}$, thus the needed bandwidth is $(N+1) r_{1}$. In general we can say that the generic term $a_{j}$ represents the probability that the $N$ streams exploit a bandwidth given by $(N+j) r_{1}$.

\section{II.3 BANDWIDTH ESTIMATION FOR HETEROGENEOUS STREAMS EXPLOITING CORRELATION AMONG STREAMS}

In this section the aggregate bandwidth under the hypothesis of a certain degree of correlation among video streams will be calculated. In particular, we define the vector $\left(\alpha_{1}, \alpha_{2}, \ldots \alpha_{N}\right)$ of the $\mathrm{N}$ bit rates relative to the video streams and suppose that there is only a correlation between two video streams of the same type, generically expressed by the following conditional probability:

$$
p\left(\alpha_{i} \mid \alpha_{j}\right)
$$

where $\alpha_{i}$ and $\alpha_{j}$ are two indices representing, respectively, the stream rates $i$ and $j$ of the same type of video stream.

Given this assumption, we can rewrite the state probability $p\left(\alpha_{1}, \alpha_{2}, \ldots \alpha_{N}\right)$ that considers generically $\mathrm{N}$ video streams of $\mathrm{M}$ different types: 


$$
p\left(\alpha_{1}, . ., \alpha_{N}\right) \approx p\left(\alpha_{1} \mid \alpha_{2}\right) . . p\left(\alpha_{N-1} \mid \alpha_{N}\right) p\left(\alpha_{N}\right)
$$

Obviously, $p\left(\alpha_{i} \mid \alpha_{j}\right)=p\left(\alpha_{i}\right)$ when $\alpha_{i}$ and $\alpha_{j}$ belong to different types of video streams. Since each term $\alpha_{i}$ expresses the $i^{\text {th }}$ bit rate, but referred to a particular type of video stream, the notation of the conditional probabilities can be usefully changed as follows:

$$
p\left(\alpha_{i} \mid \alpha_{j}\right)=p\left(f_{r_{\alpha_{i}}}^{m_{i}} \mid f_{r_{\alpha_{j}}}^{m_{j}}\right)
$$

where $p\left(f_{r_{\alpha_{i}}}^{m_{i}} \mid f_{r_{\alpha_{j}}}^{m_{j}}\right)$ represents the probability that the $m_{i}^{\text {th }}$ type of film has bit rate $r_{\alpha_{i}}$ given that the $m_{j}^{\text {th }}$ type of film has bit rate $r_{\alpha_{j}}$. To calculate the state probability we have to consider all combinations of all $\mathrm{N}$ video streams of $\mathrm{M}$ different types that give the considered state of the system, but weighting them by the probability that a generic user chooses the $m^{\text {th }}$ type of film, represented generically by the factor $\beta_{m}$. The state probability assumes the expression:

$$
p\left(\alpha_{1}, \ldots, \alpha_{N}\right)=\sum_{m_{l}=1}^{M} \beta_{m_{l}} p\left(f_{r_{\alpha_{l}}}^{m_{I}}\right) \prod_{i=2}^{N}\left(\sum_{m_{i}=1}^{M} \beta_{m_{i}} p\left(f_{r_{\alpha_{i}}}^{m_{i}} \mid f_{r_{\alpha_{i-l}}}^{m_{i-1}}\right)\right)
$$

Assuming all the histogram bins of all the video streams of the same size (i.e. $r_{k}=k r_{1}$ for each $1 \leq k \leq K$ and for each type of video stream), it is intuitive to realize that the total bandwidth occupied by all the video streams will be proportional to the basic bin size $r_{1}$. We can then define the quantity $a_{h}$, which represents the probability of the aggregation of $\mathrm{N}$ video streams occupying a bandwidth of $h r_{1}$. It will result:

$$
a_{h}=\sum_{r_{1}+r_{2}+. .+r_{N}=h r_{1}} p\left(\alpha_{1}, \ldots, \alpha_{N}\right)
$$

To find the probability $P\left(\Lambda \leq \Lambda_{S}\right)$ we have to sum the probabilities of a bandwidth $h r_{l}$ (say, the terms $a_{h}$ ), but only if $h r_{1} \leq \Lambda_{S}$. Considering that the minimum value of the parameter $h$ is $N$ (corresponding to the case in which all the video streams are in their minimum bit rate $r_{1}$ ), it results:

$$
P\left(\Lambda \leq \Lambda_{S}\right)=\sum_{h=N}^{\Lambda_{S} / r_{l}} a_{h}
$$

where, remembering (20) and (21):

$$
a_{h}=\sum_{r_{1}+\ldots+r_{N}=h r_{l}} \sum_{m_{l}=1}^{M} \beta_{m_{l}} p\left(f_{r_{I}}^{m_{I}}\right) \prod_{i=2}^{N}\left(\sum_{m_{i}=1}^{M} \beta_{m_{i}} p\left(f_{r_{i}}^{m_{i}} \mid f_{r_{i-1}}^{m_{i-l}}\right)\right)
$$

To find the aggregate bandwidth, the first step to perform is the calculation of the terms $a_{h}$ through (21), but a direct approach is computationally very expensive. To find a simpler iterative solution, let us make the following position: 


$$
g_{r_{N-l}, m_{N-l}, h_{l}}^{(1)}=\sum_{m_{N}=1}^{M} \beta_{m_{N}} p\left(f_{r_{N}}^{m_{N}} \mid f_{r_{N-l}}^{m_{N-l}}\right)_{r_{N}=h_{l}}
$$

The function $g_{r_{N-1}, m_{N-1}, h_{l}}^{(1)}$ is a matrix with three dimensions, since the parameters are $r_{N-1}, m_{N-1}$ and $h_{l}$. The following limitations are valid:

$$
\begin{gathered}
1 \leq r_{N-1} \leq K \\
1 \leq h_{1} \leq K \\
1 \leq m_{N-1} \leq M
\end{gathered}
$$

since the bit rate $r_{N-1}$ and $r_{N}$, normalized to the basic bin size $r_{1}$, can assume $\mathrm{K}$ integer values (and so also $h_{l}$ can assume the same values of $r_{N}$ ), while $m_{N-l}$ represents the type of the video stream. Now let us consider the function:

$$
g_{r_{N-2}, m_{N-2}, h_{l}}^{(2)}=\sum_{r_{N}+r_{N-l}=h_{l}} \prod_{i=N-1}^{N} \sum_{m_{i}=1}^{M} \beta_{m_{i}} p\left(f_{r_{i}}^{m_{i}} \mid f_{r_{i-1}}^{m_{i-1}}\right)
$$

that is the sum of all the combinations of the product of two factors like $g_{r_{N-1}, m_{N-1}, h_{l}}^{(1)}$, but verifying the condition under the external summation notation (that is, the sum of the rates $r_{N}$ and $r_{N-1}$ has to be equal to $h_{l}$ ). Consequently, the limitations of the parameters of $g_{r_{N-2}, m_{N-2}, h_{l}}^{(2)}$ are:

$$
\begin{gathered}
1 \leq r_{N-2} \leq K \\
2 \leq h_{1} \leq 2 K \\
1 \leq m_{N-2} \leq M
\end{gathered}
$$

and analyzing the structure of $g_{r_{N-2}, m_{N-2}, h_{l}}^{(2)}$ we note that:

$$
g_{r_{N-2}, m_{N-2}, h_{l}}^{(2)}=\sum_{r_{N-l}=1}^{K}\left[\sum_{m_{N-l}=1}^{M} \beta_{m_{N-l}} p\left(f_{r_{N-1}}^{m_{N-1}} \mid f_{r_{N-2}}^{m_{N-2}}\right) g_{r_{N-1}, m_{N-1}, h_{l}-r_{N-l}}^{(1)}\right]
$$

Now let us analyze the function:

$$
g_{r_{N-3}, m_{N-3}, h_{l}}^{(3)}=\sum_{r_{N}+r_{N-1}+r_{N-2}=h_{l}} \prod_{i=N-2}^{N} \sum_{m_{i}=1}^{M} \beta_{m_{i}} p\left(f_{r_{i}}^{m_{i}} \mid f_{r_{i-l}}^{m_{i-1}}\right)
$$

Making the same considerations of $g_{r_{N-2}, m_{N-2}, h_{l}}^{(2)}$, we can easily find that:

$$
g_{r_{N-3}, m_{N-3}, h_{l}}^{(3)}=\sum_{r_{N-2}=1}^{K}\left[\sum_{m_{N-2}=1}^{M} \beta_{m_{N-2}} p\left(f_{r_{N-2}}^{m_{N-2}} \mid f_{r_{N-3}}^{m_{N-3}}\right) g_{r_{N-2}, m_{N-2}, h_{l}-r_{N-2}}^{(2)}\right]
$$

and adopting the same procedure, we can find the following general iterative formula:

$$
g_{r_{N-n}, m_{N-n}, h_{l}}^{(n)}=\sum_{r_{N-n+l}=l}^{K}\left[\sum_{m_{N-n+l}=1}^{M} \beta_{m_{N-n+l}} p\left(f_{r_{N-n+l}}^{m_{N-n+1}} \mid f_{r_{N-n}}^{m_{N-n}}\right) g_{r_{N-n+l}, m_{N-n+l}, h_{l}-r_{N-n+l}}^{(n-1)}\right]
$$

This formula is valid for $2 \leq n \leq N-1$. To obtain the terms $a_{h}$, for each value of $h$, we can write: 


$$
a_{h}=\sum_{r_{l}=l}^{K}\left[\sum_{m_{l}=1}^{M} \beta_{m_{l}} p\left(f_{r_{l}}^{m_{l}}\right) g_{r_{l}, m_{l}, h-r_{l}}^{(N-1)}\right]
$$

with $N \leq h \leq K N$. In this way, we can obtain all the terms $a_{h}$ making use of the iterative method, computationally more efficient than the direct approach.

\section{NUMERICAL RESULTS}

In this section, the algorithms previously developed are applied to specific cases, in order to evaluate the aggregate bandwidth needed by a given number of streams. In particular, to evaluate the aggregate bandwidth in the case of homogeneous streams, we have considered 40000 frames of the film Star Wars which have been smoothed with a user buffer of $1 \mathrm{MB}$. The evaluated probability histogram considers 5 bins $(\mathrm{K}=5)$ and gives the following probabilities: $p_{1}=0$; $p_{2}=0.561375 ; p_{3}=0.4113 ; p_{4}=0.0021 ; p_{5}=0.025225$, while the rate $r_{1}$ is $135330 \mathrm{bit} / \mathrm{s}$. The previous data are exploited to evaluate the aggregate bandwidth comparing the results of the proposed approximations based on iterative methods with those obtained by Chernoff bound and simulation (with the starting points of the video streams uniformly distributed into the entire duration of the Star Wars film) for various values of main system parameters, namely, number of histogram bins and number of video streams, as can be seen in figures 3 and 4 . The reader is referred to reference [8] for details about the Chernoff bound.

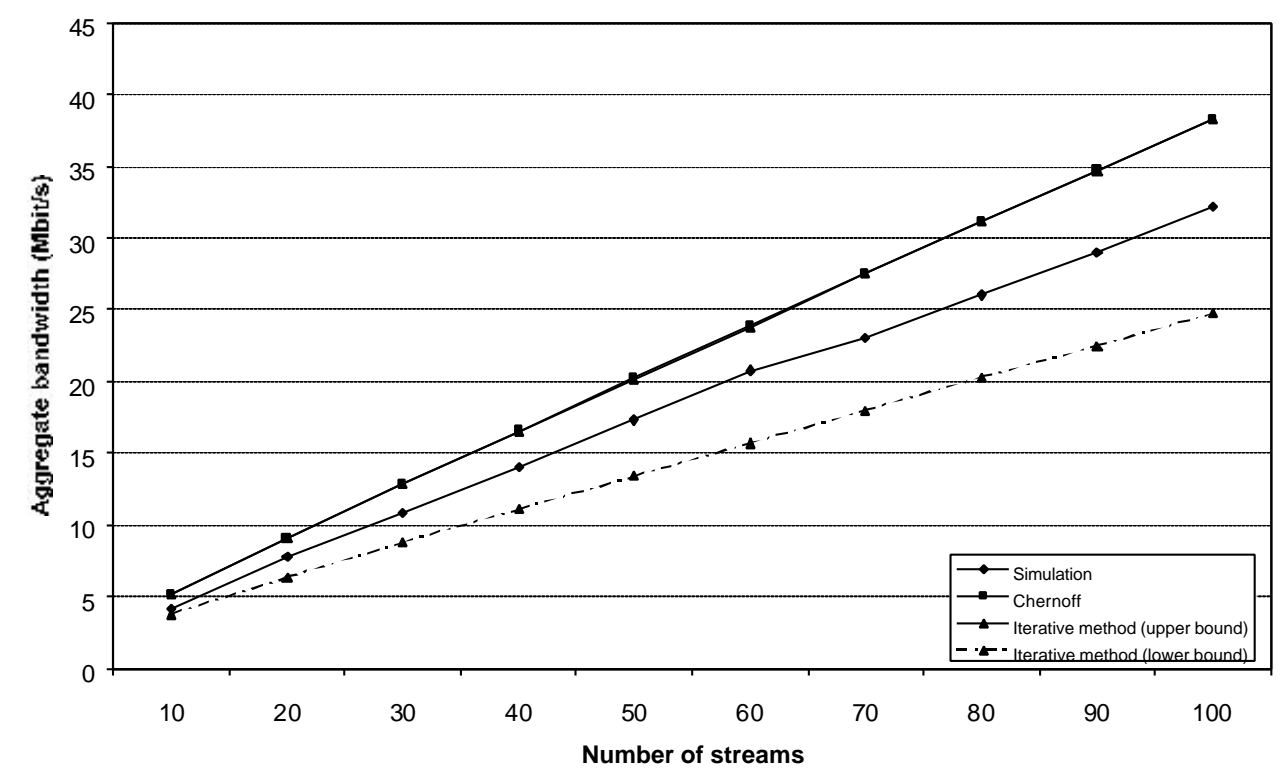

Figure 3. Aggregate bandwidth for Star Wars with a loss probability of $10^{-6}$. 


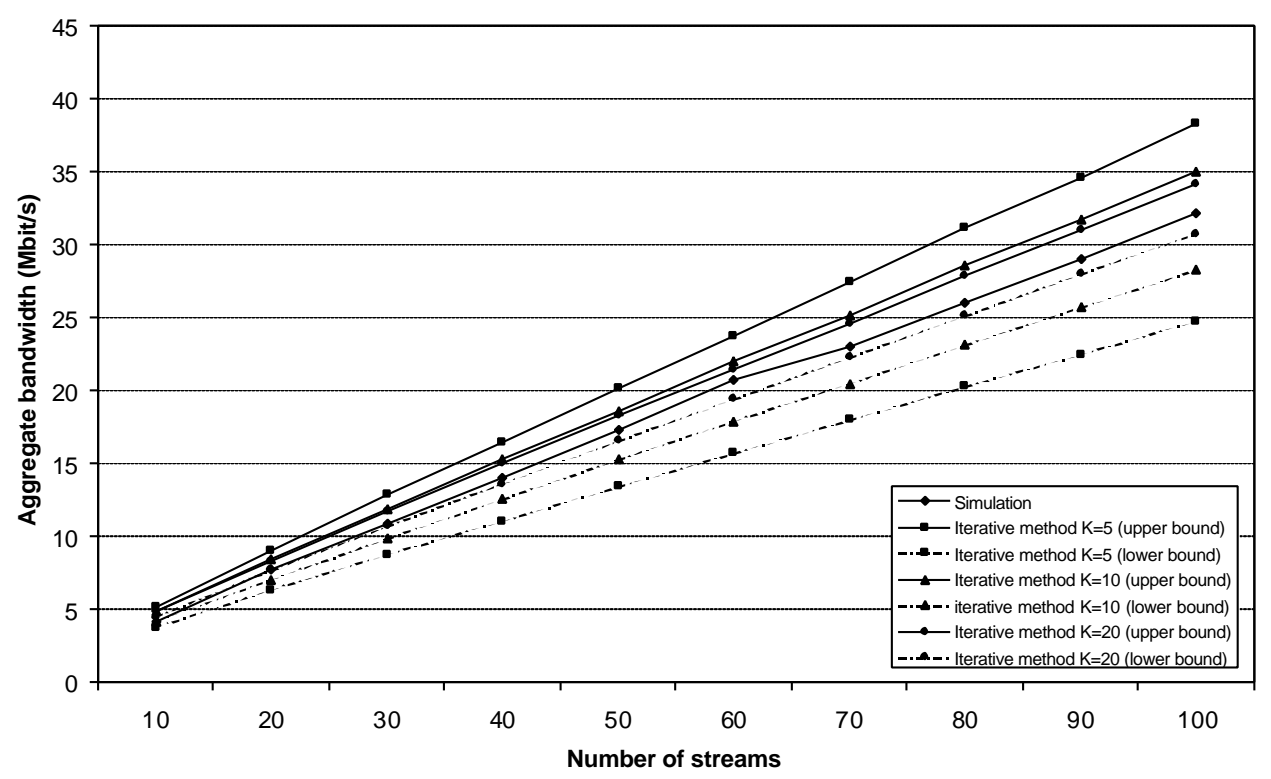

Figure 4. Aggregate bandwidth for Star Wars with a loss probability of $10^{-6}$.

As can be noted from Figure 3 the results based on the iterative method have been calculated in two ways (indicated by the continuous line and the corresponding dashed line). In the first case, all the bit rates of the frames falling into each of the histogram bins have been identified with the upper bound of their corresponding bin (continuous line), while in the second case they have been identified with the lower bound of their corresponding bin (dashed line). The curves obtained taking into account upper and lower bounds of the histogram bins define a conical area. As will be shown in the sequel of the paper, usually the simulation curve falls into this area. We can assume that the aggregate bandwidth calculated using the upper bounds of the histogram bins represents a conservative estimation of the needed bandwidth. On the other hand, if the simulation curve falls under this area, the aggregate bandwidth estimated with the algorithm will be considerably higher than the simulated one, testifying an over-conservative estimation. Such a case can happen due to the approximations introduced by the independence assumption. However, in our simulation experiments it was experienced only in some minor contexts. If, instead, the simulation curve falls above the specified area, which was not experienced in our simulation experiments, the aggregate bandwidth obtained with the algorithm will not be able to satisfy the QoS specifications, leading, in all likelihood, to a service failure.

From Figure 3 we can also note that the results obtained by the analytical method are very close to the results obtained by the Chernoff method. Also in Figure 4 the analytical method has been developed considering upper and lower bounds of the histogram bins, but it has been implemented using different numbers of bins. In particular, we can note that if the number of histogram bins increases, the conical area reduces and considerations about the position of the simulation curve, 
when compared to the conical area, can be made with greater precision. In any case, the results of the analytical method become close enough to the simulation results if the number of histogram bins increases, although after a given value there is a saturation without any further significant improvements.

In the case of heterogeneous streams, to evaluate the aggregate bandwidth we have considered 40000 frames of the films Star Wars, Asterix and a Soccer Final. They have been smoothed with a user buffer of $1 \mathrm{MB}$. The evaluated probability histogram considers 5 bars $(\mathrm{K}=5)$ with the following probabilities:

\begin{tabular}{l|l|l} 
Star Wars & Asterix & Soccer \\
\hline$p_{1}=0.179625$ & $p_{1}=0$ & $p_{1}=0$ \\
$p_{2}=0.79365$ & $p_{2}=0.001275$ & $p_{2}=0$ \\
$p_{3}=0.0024$ & $p_{3}=0.439575$ & $p_{3}=0.000375$ \\
$p_{4}=0.024325$ & $p_{4}=0.457575$ & $p_{4}=0.735025$ \\
$p_{5}=0$ & $p_{5}=0.101575$ & $p_{5}=0.2646$
\end{tabular}

The value of $r_{1}$, common to all considered films, is $212562 \mathrm{bit} / \mathrm{s}$. Moreover, we have assumed that each of these types of video streams can be chosen with the same probability $\left(\beta_{i}=1 / 3\right)$.

The previous data are exploited to evaluate the aggregate bandwidth, comparing the results of the proposed method with those obtained by simulation for various values of main system parameters, namely, number of bins and number of streams.

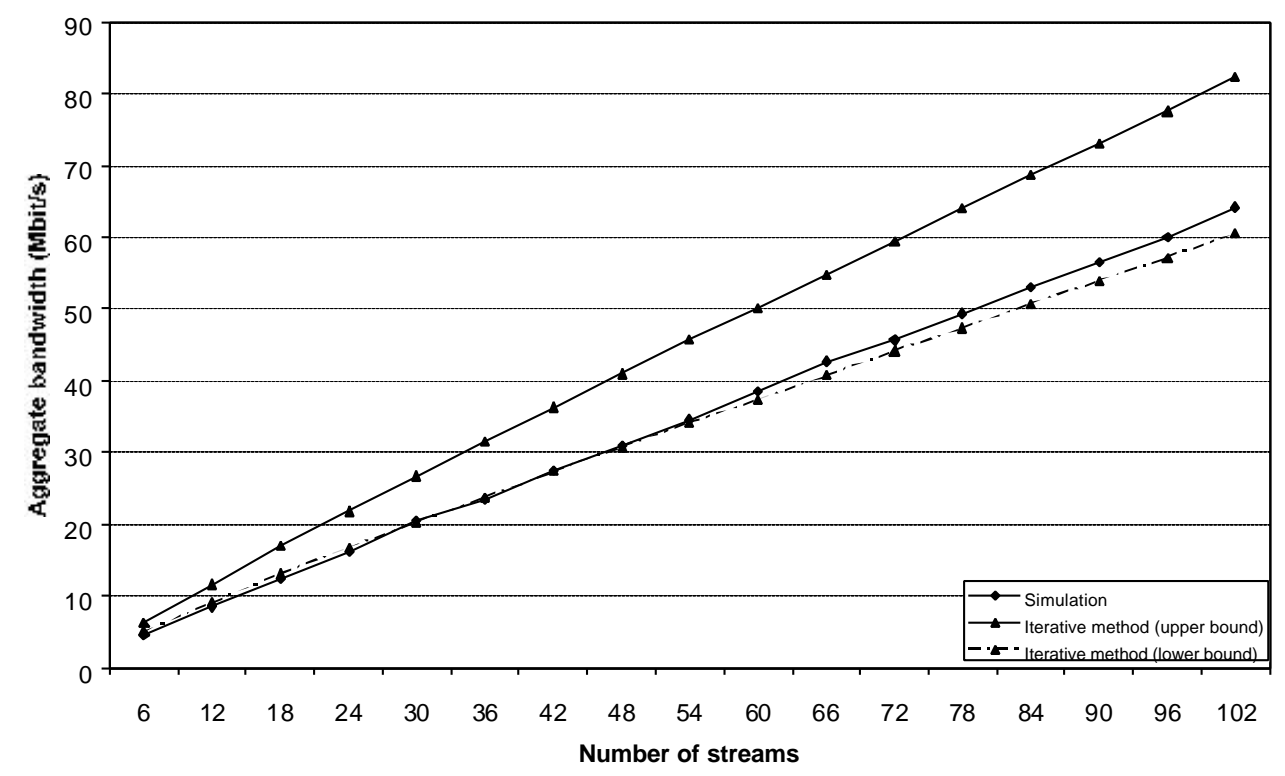


Figure 5. Aggregate bandwidth for the three films with a loss probability of $10^{-6}$.

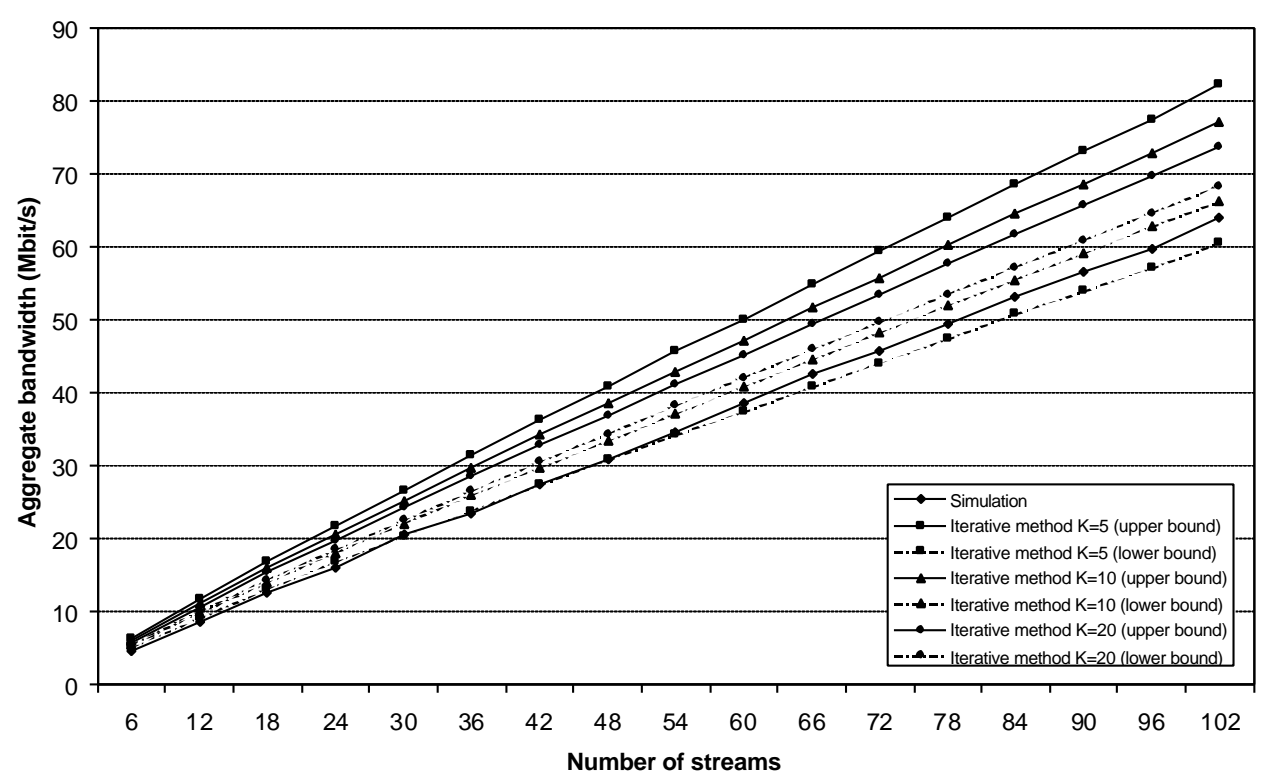

Figure 6. Aggregate bandwidth for the three films with a loss probability of $10^{-6}$.

Observing figure 5, we can note that the simulation curve is closer to the results of the iterative method using lower bounds of the histogram bins than the results of the iterative method with upper bounds. Thus the iterative method in this case is more conservative than the case of the single type of film (see also Figure 3 for a comparison between the two cases). This consideration is further justified in Figure 6, where a different number of bins have been considered. We can see that if the number of bins increases, the conical area reduces, but the simulation results fall under this area (see the results for $\mathrm{K}=10$ and $\mathrm{K}=20$ ). Furthermore, if the number of bins increases the analytical results become close enough to the simulation results, although after a certain number of bins there is a saturation without any further improvement.

To test the effectiveness of the algorithm that makes use of conditional probabilities, we have used 40000 frames of the three films Star Wars, Asterix and a Soccer Final. As before, we have assumed that each of these types of video stream can be chosen with the same probability $\left(\beta_{i}=1 / 3\right)$. The total bit rate interval of all the types of video streams has been divided first into 15 equal bins of size $\Delta=70854 \mathrm{bit} / \mathrm{s}$ and then into 20 equal bins of size $\Delta=53141 \mathrm{bit} / \mathrm{s}$. The loss probability has been assumed as $10^{-6}$. All the bandwidth results have been obtained by supposing that all video streams start into a time interval of 1800 frames (corresponding to one minute). In Figure 7 there is a comparison between the results that make use of the conditional probabilities, independent probabilities and the simulation results. As can be noted the upper and lower bounds are quite close together and simulation results usually lie between the two bounds. In addition, lower bounds 
obtained by conditional probabilities prove to be closer to simulation than upper bound, confirming the usefulness of the approach.

Figure 8 shows the same results obtained in Figure 7, but with 20 histogram bins. In this case, the marginal distribution of the video streams using the histogram method is better described, giving results closer to the simulation, but the same considerations as Figure 7 can be made.

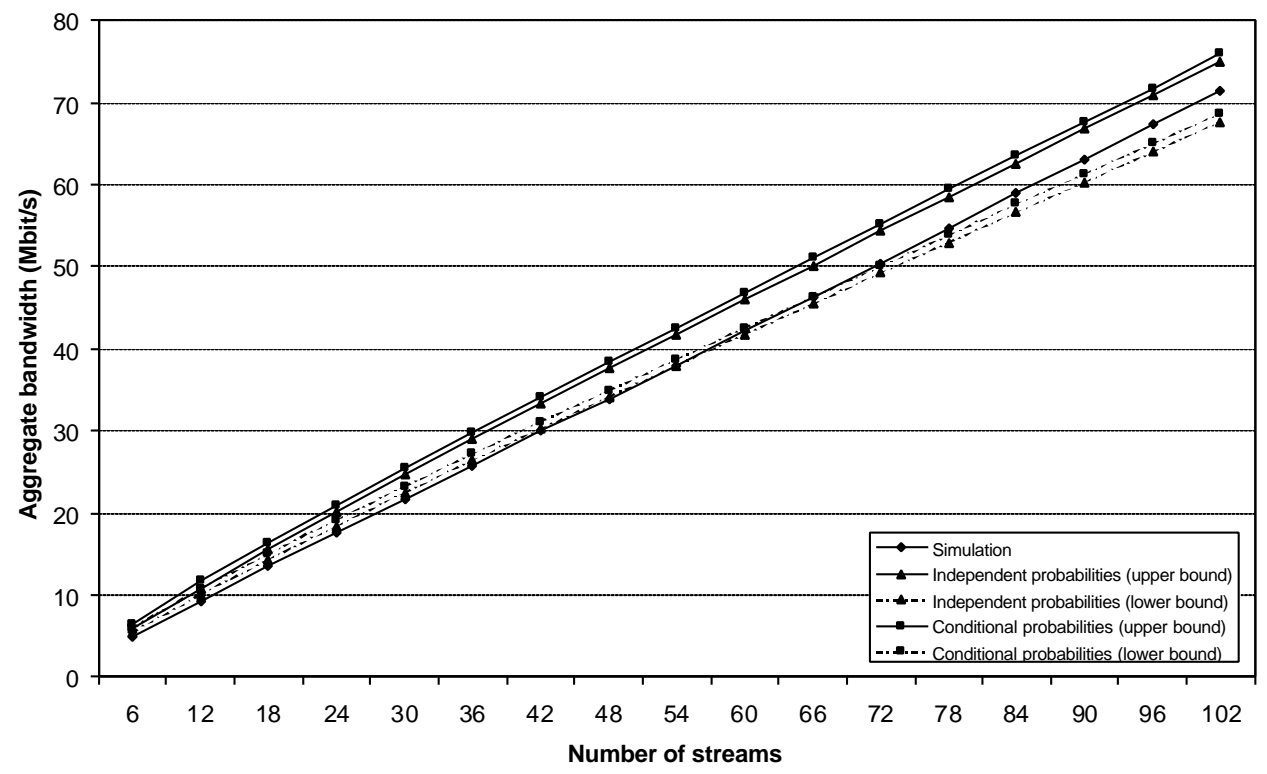

Figure 7. Aggregate bandwidth for three films with a loss probability of $10^{-6}$ and 15 histogram bins. The starting points of all streams are uniformly selected in a time interval of 1800 frames.

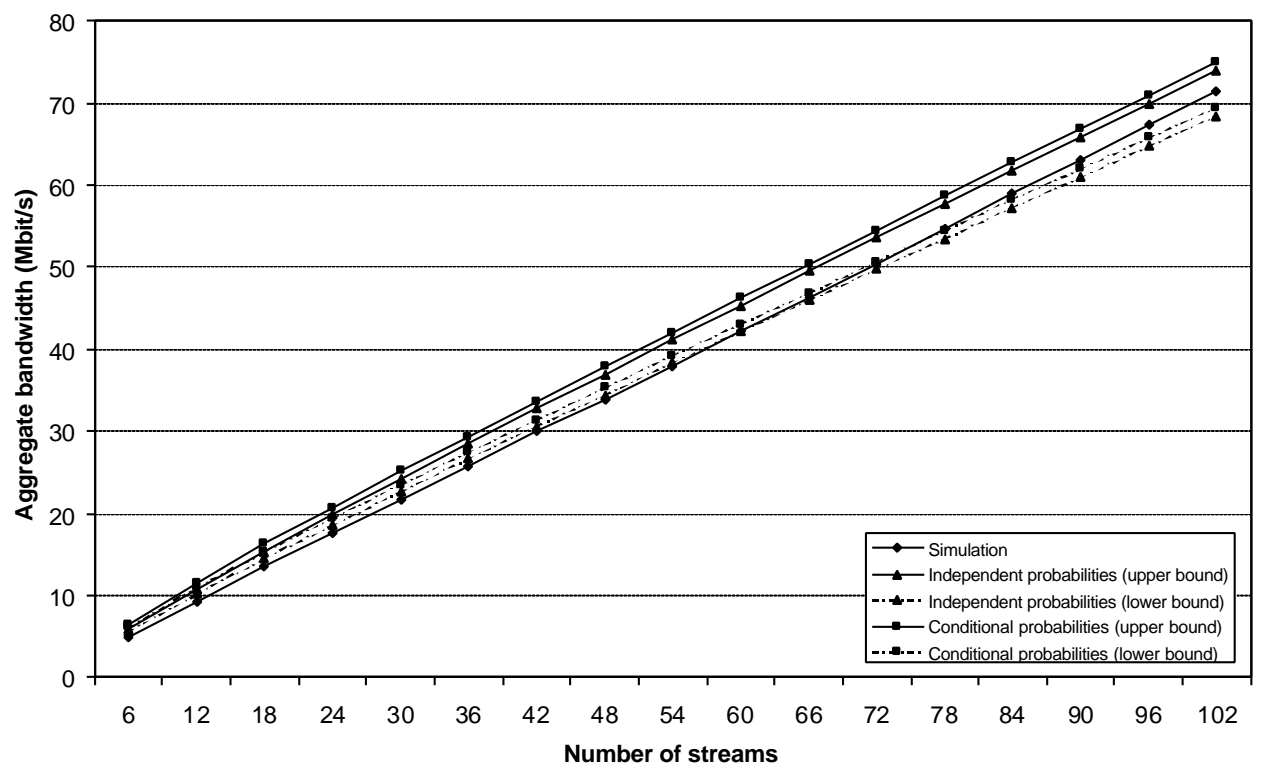

Figure 8. Aggregate bandwidth for three films with a loss probability of $10^{-6}$ and 20 histogram bins. The starting points of all streams are uniformly selected in a time interval of 1800 frames. 


\section{CONCLUSIONS}

The algorithms presented in this paper for bandwidth estimation in VBR video distribution systems represent a new approach to the problem. Such an approach proves valid independently of the shape of the rate probability distribution and can be easily used even if the distribution is not referable to those usually noted in the literature. A significant positive aspect of the proposed algorithms is the evaluation of bandwidth lower and upper bounds, obtained exploiting experimental data deriving from field measurements. The obtained results can be fruitfully and realistically exploited in designing video distribution systems.

\section{APPENDIX A}

The goal of this appendix is to present an iterative method for evaluating the bandwidth needed by a given number $N$ of independent video streams. Recalling equation (8), we know that:

$$
p\left(\Lambda \leq \Lambda_{S}\right) \leq\left(\sum_{j=N}^{h \max }\left(\sum_{\begin{array}{c}
n 1+n 2+\ldots+n K=N \\
(n 1+2 n 2+\ldots+K n K)=j
\end{array}} N ! \prod_{i=1}^{K} \frac{p_{i}{ }^{n i}}{n i !}\right)\right)
$$

Let us make the following position:

$$
a_{j-N}=\sum_{\substack{n I+n 2+\ldots+n K=N \\(n 1+2 n 2+\ldots+K n K)=j}} N ! \prod_{i=1}^{K} \frac{p_{i}{ }^{n i}}{n i !} \quad \forall 0 \leq j-N \leq(K-1) N
$$

The first term of the sum is obtained for $n_{1}=N$ and $n_{2}=n_{3}=. .=n_{K}=0$ (and so $j=N$ ). The value of this term is: $a_{0}=p_{1}^{N}$ and clearly represents the probability that all streams are present in the network with their minimum rate $r_{l}$.

Similarly, the second addend of the external sum of (A.1) will be obtained assuming $n_{1}=N-1, n_{2}=1$ and $n_{3}=. .=n_{K}=0$ (and so $j=N+1$ ). We have:

$$
a_{1}=N !\left(p_{1}^{N-1} p_{2}\right) /(N-1) !=N p_{1}^{N-1} p_{2}
$$

and therefore:

$$
p_{1} a_{1}=N p_{1}^{N} p_{2}=N p_{2} a_{0} .
$$

Now let us find the term $a_{2}$. The conditions to be respected are the following:

$$
\left\{\begin{array}{c}
n_{1}+2 n_{2}+\ldots+K n_{K}=N+2 \\
n_{1}+n_{2}+\ldots+n_{K}=N
\end{array}\right.
$$


There are two cases that respect the conditions mentioned above. The first is $n_{1}=N-2, n_{2}=2$ and $n_{3}=. .=n_{K}=0$ and the second is $n_{1}=N-1, n_{3}=1$ and $n_{2}=n_{4}=. .=n_{K}=0$. For this reason, the term $a_{2}$ will be the sum of two addends and can be written as follows:

$$
a_{2}=\frac{N(N-1) p_{1}^{N-2} p_{2}^{2}}{2}+N p_{1}^{N-1} p_{3} .
$$

Consequently:

$$
p_{1} a_{2}=[(N-1) / 2] p_{2} a_{1}+N p_{3} a_{0} .
$$

If we now observe the structure of the terms $p_{1} a_{1}$ and $p_{1} a_{2}$, we can also suppose the structure of $p_{1} a_{3}$. Let us imagine that the term $p_{1} a_{3}$ is structured in this way:

$$
p_{1} a_{3}=\frac{N-2}{3} p_{2} a_{2}+C_{1}(N) p_{3} a_{1}+C_{2}(N) p_{4} a_{0}
$$

where $C_{1}(N)$ and $C_{2}(N)$ are generic coefficients depending only on the number $\mathrm{N}$ of flows. To find the value of these coefficients, let us consider the previous expressions $a_{2}, a_{1}$ and $a_{0}$. We find that:

$$
a_{3}=\frac{N(N-1)(N-2)}{6} p_{1}^{N-3} p_{2}^{3}+\frac{N(N-2)}{3} p_{1}^{N-1} p_{2}+N C_{1}(N) p_{1}^{N-2} p_{2} p_{3}+C_{2}(N) p_{1}^{N-1} p_{4}
$$

This expression suggests all the combinations of $n_{i}$ that verify the constraints necessary to find $a_{3}$, which are:

$$
\left\{\begin{array}{c}
n_{1}+2 n_{2}+\ldots+K n_{K}=N+3 \\
n_{1}+n_{2}+\ldots+n_{K}=N
\end{array}\right.
$$

In fact, the first addend of $a_{3}$ suggests that $n_{1}=N-3, n_{2}=3$ and $n_{3}=. .=n_{K}=0$; the second and the third addend suggest that $n_{1}=N-2, n_{2}=n_{3}=1$ and $n_{4}=. .=n_{K}=0$; the fourth suggests that $n_{1}=N-1, n_{4}=1$ and $n_{2}=n_{3}=n_{5} . .=n_{K}=0$. We can easily observe that all these combinations of $n_{i}$ verify the two constraints described above.

We can then calculate the exact expression of $a_{3}$ through the $n_{i}$ combinations found above and utilizing (A.1); the result is given by:

$a_{3}=\frac{N(N-1)(N-2)}{6} p_{1}^{N-3} p_{2}^{3}+N(N-1) p_{1}^{N-1} p_{2} p_{3}+N p_{1}^{N-1} p_{4}$. Comparing this equation with (A.2),

it is possible to find the values for $C_{1}(N)$ and $C_{2}(N)$ :

$$
C_{1}(N)=(2 N-1) / 3 ; C_{2}(N)=N
$$

So we can assert that:

$$
p_{1} a_{3}=[(N-2) / 3] p_{2} a_{2}+[(2 N-1) / 3] p_{3} a_{1}+N p_{4} a_{0}
$$


The same procedure illustrated here to find $p_{1} a_{3}$ can obviously be adopted to find all the other terms $p_{1} a_{4}, p_{1} a_{5}$, etc. In short, we have to express the generic term $p_{1} a_{j}$ in two ways: as a function of all the $C_{i}(N)$ and considering all the combinations of $n_{i}$. Then, comparing the two expressions, we derive all the $C_{i}(N)$.

By way of illustration, we report the term $p_{1} a_{4}$ found applying this method:

$$
p_{1} a_{4}=\frac{N-3}{4} p_{2} a_{3}+\frac{3 N-3}{6} p_{3} a_{2}+\frac{3 N-1}{4} p_{4} a_{1}+N p_{5} a_{0}
$$

In general, since the probabilities are in number of $K$ (that is the number of the bins of the histogram), for $j$ from 1 to $K-1$, the terms $p_{1} a_{j}$ will present addends containing the terms $a_{j-i}$ to the term $a_{0}$, while for $j>K-1$, all the terms $p_{1} a_{j}$ will contain exactly $K$ - 1 addends, each of them containing the probabilities from $p_{2}$ to $p_{K}$.

It is possible to directly find all the coefficients, $C_{i}^{j}(N)$, of the generic term $p_{1} a_{j}$ with a simple expression. In particular we can note that in general:

$$
C_{i}^{j}(N)=\left[\left(\begin{array}{l}
j-1 \\
i-1
\end{array}\right) N-\left(\begin{array}{c}
j-1 \\
i
\end{array}\right)\right] /\left(\begin{array}{l}
j \\
i
\end{array}\right)
$$

for $3 \leq i \leq j-1$ and $4 \leq j \leq N(K-1)$. After some simple algebra we obtain:

$$
C_{i}^{j}(N)=\frac{(N+1) i}{j}-1
$$

(A.3) presents the advantages of great simplicity and it is also independent of the coefficients $C_{i}^{j-1}(N)$ calculated in the previous step. Using (A.3) in the general formula, we find the expression:

$$
p_{1} a_{j}=\sum_{i=1}^{K-1}\left[\frac{(N+1) i}{j}-1\right] p_{i+1} a_{j-i}
$$

with

$$
\left\{\begin{array}{c}
j=1, \ldots,(K-1) N \\
a_{j-i}=0 \text { if } j-i<0
\end{array} .\right.
$$




\section{REFERENCES}

[1] Andrew s. Tanenbaum, Computer Networks, Prentice-Hall, Third Edition 1996.

[2] Mark W. Garrett, Walter Willinger, "Analysis, Modeling and Generation of Self-Similar VBR Video Traffic" ACM Computer Communications Review, Vol. 24, No. 4, pp. 269-280, October 1994.

[3] R. G. Garroppo, S. Giordano, S. Miduri, M. Pagano, F. Russo, "Statistical Multiplexing of SelfSimilar VBR Videoconferencing Traffic", Proc. of IEEE GLOBECOM '97, Phoenix, November 1997.

[4] Predrag R. Jelenkovic, Aurel A. Lazar, Nemo Semret, "The Effect of Multiple Time Scales and Subexponentiality in MPEG Video Streams on Queuing Behavior", IEEE Journal on Selected Area in Communications, vol. 15, Nº 6, pp. 1052-1071, August 1997.

[5] Aurel A. Lazar, Giovanni Pacifici, Dimitrios E. Pendarakis, "Modeling Video Sources for RealTime Scheduling”, Multimedia Systems, vol. 1, Nº 6, pp. 253-266, April 1994.

[6] Matthias Grossglauser, David Tse, "A Framework for Robust Measurement-Based Admission Control", IEEE / ACM Transactions on Networking, vol. 7, N 3, pp. 293-309, June 1999.

[7] Edward W. Knightly, Ness B. Shroff, "Admission Control for Statistical QoS: Theory and Practice", IEEE Network, vol. 13, N² 2. pp. 20-29, March 1999.

[8] Zhi-Li Zhang, Jim Kurose, James D. Salehi, Don Towsley, "Smoothing, Statistical Multiplexing, and Call Admission Control for Stored Video", IEEE Journal on Selected Areas in Communications, vol. 15, Nº 6, pp. 1148-1166, August 1997.

[9] James D. Salehi, Zhi-Li Zhang, Jim Kurose, Don Towsley, "Supporting Stored Video: Reducing Rate Variability and End-to-End Resource Requirements Through Optimal Smoothing”, IEEE/ACM Transactions On Networking, vol. 6, N N $^{\circ}$, pp. 397-410, August 1998.

[10] Wu-chi Feng, Jennifer Rexford, "Performance Evaluation of Smoothing Algorithms for Transmitting Prerecorded Variable-Bit-Rate Video", IEEE Transactions on Multimedia, pp. 302-313, September 1999.

[11] San-qi Li, Song Chong, Chia-Lin Hwang, "Link Capacity Allocation and Network Control by Filtered Input Rate in High Speed Networks", IEEE/ACM Transactions on Networking, vol. 3, $\mathrm{N}^{\mathrm{o}}$ 1, pp. 10-25, Feb. 1995.

[12] Chia-Lin Hwang, San-qi Li, "On Input State Space Reduction and Buffer Noneffective Region”, In Proc. IEEE INFOCOM'94, pp. 1018-1028, June 1994.

[13] Daniel P. Heyman, T.V. Lakshman, "What Are the Implications of Long-Range Dependence for VBR-Video Traffic Engineering?", IEEE/ACM Transactions on Networking, vol.4, $\mathrm{N}^{\mathrm{o}} 3$, pp. 301-317, June 1996.

[14] Marwan Krunz, Herman Hughes, "A Traffic Model for MPEG-Coded VBR Streams", In Proc ACM SIGMETRICS, Ottawa, Canada, vol. 23, Nº 1, pp. 47-55, May 1995.

[15] Paul Skelly, Mischa Schwartz, Sudhir Dixit, "A Histogram-Based Model for Video Traffic Behavior in an ATM Multiplexer", IEEE/ACM Transactions on Networking, vol. $1, \mathrm{~N}^{\circ} 4$, pp. 446-458, August 1993.

[16] D. Striccoli , 2000. "Stima della banda aggregata per sistemi di distribuzione video", Dr. Eng. Degree Thesis, Polytechnic of Bari, Italy (in Italian). 